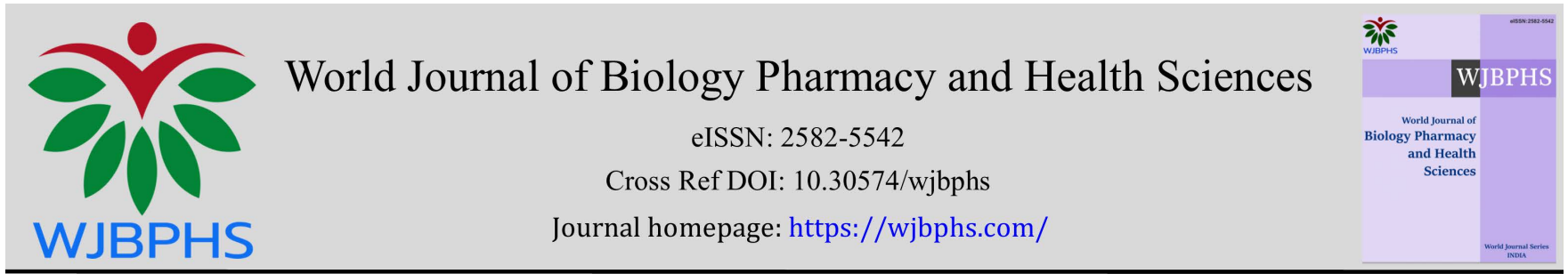

(RESEARCH ARTiCLE)

\title{
Thin layer chromatographic investigation of antibiotics residues in edible poultry tissues in Bangladesh
}

\author{
Md. Shafiqul Islam *, Md. Rakibul Hasan, Md. Shakil Islam \\ Department of Pharmacology, Faculty of Veterinary Science, Bangladesh Agricultural University, Mymensingh-2202, \\ Bangladesh.
}

World Journal of Biology Pharmacy and Health Sciences, 2021, 05(03), 024-032

Publication history: Received on 27 January 2021; revised on 26 February 2021; accepted on 01 March 2021

Article DOI: https://doi.org/10.30574/wjbphs.2021.5.3.0019

\begin{abstract}
Majority of the people in Bangladesh are still not aware about the health hazards of antibiotic residues. In this perspective, a survey was undertaken at five different poultry farms in the Dinajpur district. One hundred liver, one hundred thigh muscle and one hundred breast muscle samples were collected from different farms \& market places and evaluated by TLC method. A very negligible number of samples were found positive for antibiotics residues. Out of one hundred liver, one hundred thigh muscle and one hundred breast muscle samples respectively; only two liver, two breast muscle and two thigh muscle samples were found positive for amoxicillin antibiotic; five liver, three breast muscle and three thigh muscle samples were found positive for ciprofloxacin; three liver, two breast muscle and two thigh muscle samples were found positive for cephalexin; two liver, two breast muscle and two thigh muscle samples were found positive for enrofloxacin; four liver, two breast muscle and two thigh muscle samples were found positive for oxytetracycline. Gentamicin and neomycin were found negative for any samples. Further investigation was done in indoor discriminate and indiscriminate use of antibiotics (ciprofloxacin and oxytetracycline) in broilers. Liver, thigh muscle and breast muscle samples were collected and evaluated by TLC method. Therefore, poultry treated with antibiotics are required for specific withdrawal period until all residues are depleted to safe levels before human consumption. This research project provides idea that Bangladeshi poultry products are free of antibiotics residues in north area (Dinajpur District) of Bangladesh.
\end{abstract}

Keywords: Antibiotic residues; TLC; Oxytetracycline; Ciprofloxacin

\section{Introduction}

Poultry is one of the world's quickest growing sources of meat, addressing nearly one-fourth of all the meat produced during the year 2000. Now advanced production unit can produce broiler chickens in less than six weeks. This development arose from hereditary selection, improved feeding and health management practices. It involves usage of antibiotics as therapeutic agents to treat bacterial diseases in intensive farming systems [2].

In poultry industry, antibiotics are the most widely used veterinary drugs [14]. They are used by the poultry industry and veterinarians to improve growth rates, health, feed efficiency, egg production, or for therapeutic reasons and prophylaxis measures to reduce the incidence of poultry diseases [4,11]. In Bangladesh, a large number of poultry farming is done by the illiterate poultry farmers who do not care veterinary prescription and supervision and resulting indiscriminate use of antibiotics in poultry farming. Toxic level of antibiotic residues from poultry origins had caused several pathological defects in human health that are of public health significance $[3,10,12]$. Therefore, for the treatment of poultry with antibiotics require specific withdrawal period until all residues are depleted to safe levels for human consumption [7,13]. Now a days, appearance of antibiotics residue in edible animal tissues remain a global problem [6].

\footnotetext{
* Corresponding author: Md. Shafiqul Islam

Department of Pharmacology, Faculty of Veterinary Science, Bangladesh Agricultural University, Mymensingh-2202, Bangladesh. 
Healthy nation is the first priority to the development of a country. The people in Bangladesh are still not aware about the antibiotic residues and the health hazards of antibiotic residues. In fact, there is no extensive research work on the antibiotics residue detection and hazard analysis in Bangladesh. Moreover, there is no specific residue monitoring program in Bangladesh. Without ensuring our edible poultry tissues and products are free of antibiotics residue and micro-organism through proper researches and investigations, we cannot be able to export our poultry product to earn foreign currency. The project is directly concern to the national development. If we can not aware the people, in the long run, the nation will face the unlimited and irreparable loss. The indiscriminate use of antimicrobials leads to several life threatening implications. Among them, the most prevalent effects are antimicrobial resistance, antibiotics residues in food animal products which cause hypersensitivity reaction, alteration of gut micro flora and residual toxicity.

Nowadays, different analytical and screening methods are available. Chromatographic analysis like thin layer chromatography, liquid chromatography, gas chromatography and more advanced mass spectrometry have been replacing the conventional microbiological and immunological detection and quantification of antibiotic residue from food and environmental samples as these methods provide more recovery rate even several-fold higher [9]. Hence, chromatographic techniques are preferred to other analytical techniques even though they are quite expensive and sophisticated. Therefore, the proposed work was undertaken to investigate the toxic level of antibiotics residue in edible poultry tissues (liver, thigh muscle and breast muscle) \& to address the awareness campaign among farmers to avoid indiscriminate use of antibiotics in poultry production.

\section{Material and methods}

\subsection{Ethical approval of laboratory animals}

The experimental broilers were used ethically and at the end of the experiment sacrificed humanely following the ethical and welfare guidelines set by the Animal Welfare and Experimental Ethics Committee of Bangladesh Agricultural University [approval number: AWEEC/ BAU/2021(07)].

\subsection{Study design}

A preliminary survey on poultry farming was made in the north area (Dinajpur District) of Bangladesh and five commercial farms were selected for the study after making a contract with the farmers. A meeting was held in each farm to obtain data of rearing system, treatment records, name \& type of drugs used, withdrawal period maintenance etc. Awareness campaign also held in each farms to teach the poultry farmers on residual effects of antibiotics hazards for human health. After successful discussion, necessary samples (liver, thigh muscles and breast muscles) were collected for laboratory investigation. On the other hand, indoor experiment was also done on discriminate and indiscriminate use of antibiotics in broiler birds. At the end of experiments, liver, thigh muscles and breast muscles were collected \& preserved under $-20^{\circ} \mathrm{C}$ until TLC evaluations.

\subsection{Indoor antibiotics experiments on broiler birds}

35 apparently healthy day-old chicks (Cobb-500) were collected for indoor experiments. Chicks were reared for up to 30 days. On day 14th, the chicks were randomly divided into five groups namely control group (group A, n=6), discriminate ciprofloxacin antibiotic group (group B, n=6), indiscriminate ciprofloxacin antibiotic group (group C, $n=6$ ), discriminate oxytetracycline antibiotic group (group $\mathrm{D}, \mathrm{n}=6$ ) and indiscriminate oxytetracycline antibiotic group (group E, $n=6$ ). Group A was kept as untreated control and received no medicated water. Group B \& C were administered ciprofloxacin and group D and E were administered oxytetracycline antibiotics. After 7 days, at the age of day 23; antibiotic supply was stopped in the group-B and D followed by withdrawal period. In group C and E, antibiotics were continued till the day of sacrifice.

\subsection{Sacrificing \& sample collection}

At the end of experiment, birds were sacrificed and liver, breast muscles and thigh muscles were collected and preserved at $-20^{\circ} \mathrm{C}$ until examination.

\subsection{Screening of antibiotic residues}

The experiment was conducted in the Department of Pharmacology, Bangladesh Agricultural University (BAU), Mymensingh. Collected samples were brought to the Postgraduate Laboratory for investigation of antibiotic residues by Thin Layer Chromatography (TLC) technique. All chemicals used were analytical grade. 


\subsection{Thin Layer Chromatography (TLC)}

Thin layer chromatography (TLC) is a sensitive and exact-reliable method for monitoring low amount of different biological and chemicals. Thin layer chromatography (TLC) is among the most helpful instruments for following the advancement of organic chemical reactions and for measuring the pureness of organic compounds in phytochemistry and Biotechnology. Like every single chromatographic methods, TLC exploits the distinctive affinity of the analyte with the mobile and stationary phases to accomplish the partition of complex mixtures of organic molecules [8]. Illumination of antibiotics against UV light helps as a simple detector for this mean. Employment of thin-layer chromatography (TLC) to pharmaceutical and medical or clinical or biological research comprises more than $50 \%$ of the technique's total application. An important application of TLC is determination of drug residues in food.

- $\quad$ TLC plate was cut into appropriate size $(5 \times 4 \mathrm{~cm})$

- A straight line was drawn across the plate approximately $2 \mathrm{~cm}$ from the bottom by a pencil. Another straight line was drawn across the plate below $1 \mathrm{~cm}$ from the upper edge of the plate.

- Desired spots marking were marked on the bottom line where analytes were dropped.

- Spots were applied to the plate using thin capillary glass pipettes. A volume of $10 \mu \mathrm{l}$ was used for spotting.

- $\quad$ Plate was placed in TLC tank (contained mobile phase: Acetonitrile and Methanol; $1: 1$ ) and covered by lid and it was left until the mobile phase reached the upper line.

- $\quad$ Spots were visualized in UV detection box at $256 \mathrm{~nm}$

- $\quad$ Spots marking were done by pencil for calculation of retention factor (Rf)

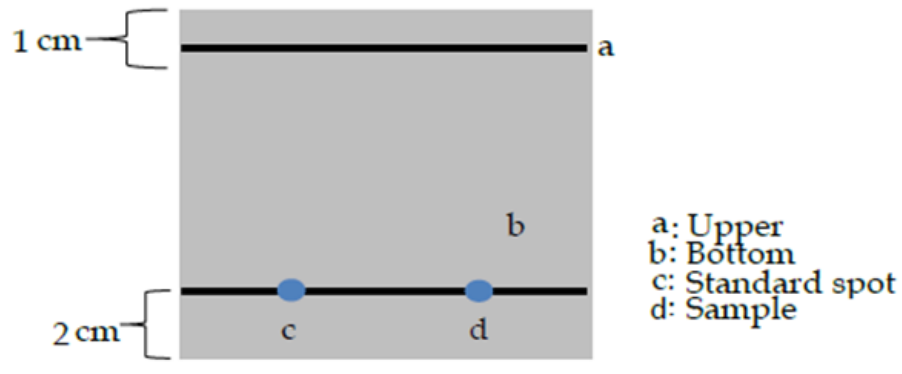

Figure 1 TLC plate

\subsection{Calculation of $R_{f}$ values}

The measurements are regularly taken from the plate to help recognize the compounds present. These estimations are the distance travelled by the solvent, and the distance went by singular spots. At the point when the solvent front draws near to the highest point of the plate, the plate is taken out from the beaker and the location of the solvent is marked with a different line before it gets an opportunity to evaporate. [8]

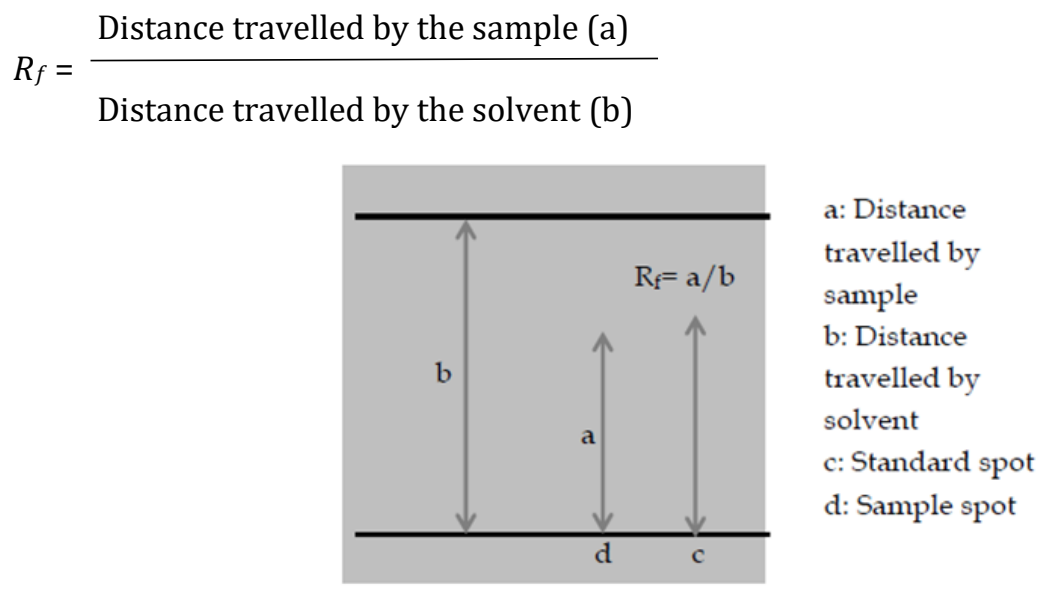

Figure 2 Calculation of Rf values 


\subsection{Interpretation of results}

In order to interpret the results, first setting of standard with reference/pure substances was determined with three repeated times of examination by standard solution. A substance was positively identified in the unknown solution when it behaved identically as the reference substance. That is, after comparison of two substances (standard \& unknown) based on following criteria a sample was positively identified such as:

- $\quad$ Same color under UV light

- $\quad$ Same Rf value as those of the reference sample.

\section{Results}

A total of one hundred livers, one hundred thigh muscles and one hundred breast muscles were collected from different farms, market places and analyzed in the Pharmacology Laboratory, Bangladesh Agricultural University, Mymensingh, Bangladesh. The antibiotics screening tests were done on the very popular \& mostly used antibiotics namely amoxicillin, ciprofloxacin, cephalexin, enrofloxacin, oxytetracycline, gentamicin and neomycin. Results were demonstrated in table 1 as well as figure 1, figure 4 and figure 5 respectively. TLC analysis revealed that out of one hundred liver samples only two samples were positive for amoxicillin, three samples were positive for ciprofloxacin, four samples were positive for cephalexin, two samples were positive for enrofloxacin, two samples were positive for tetracycline and gentamicin \& neomycin were found negative for any samples. In case of thigh muscles, out of one hundred samples, two samples were positive for amoxicillin, three samples were positive for ciprofloxacin, four samples were positive for cephalexin, two samples were positive for enrofloxacin, two samples were positive for tetracycline and gentamicin \& neomycin were found negative for any samples. In case of breast muscles, out of one hundred samples, two samples were positive for amoxicillin, three samples were positive for ciprofloxacin, four samples were positive for cephalexin, two samples were positive for enrofloxacin, two samples were positive for tetracycline and gentamicin \& neomycin were found negative for any samples. On the other hands, discriminate and indiscriminate use of ciprofloxacin and oxytetracycline antibiotics experiments were also done and analyzed. Samples such as liver, breast muscles and thigh muscles were collected and analyzed by TLC techniques. Control and discriminate groups of birds were found negative for any antibiotics as shown in table 2, table 3 and figure 6 respectively. However, indiscriminate antibiotics group birds demonstrated antibiotics residues positive in liver, thigh muscle and breast muscle. Out of six samples for each; six liver, four thigh muscles and four breast muscles were found positive for ciprofloxacin and five liver, four thigh muscles and four breast muscles were found positive for oxytetracycline and results were demonstrated in table 2, table 3 and figure 6.

Table 1 Antibiotics residues

\begin{tabular}{|l|l|l|l|l|}
\hline \multirow{2}{*}{ Name of samples } & Liver, $\mathbf{n = 1 0 0}$ & $\begin{array}{l}\text { Thigh muscles } \\
\mathbf{n = 1 0 0}\end{array}$ & $\begin{array}{l}\text { Breast muscles } \\
\mathbf{n = 1 0 0}\end{array}$ & Rf value \\
\cline { 2 - 4 } & Prevalence \% & Prevalence \% & Prevalence \% & \\
\hline Amoxicillin & $2 \%$ & $2 \%$ & $2 \%$ & Same with std. \\
\hline Ciprofloxacin & $5 \%$ & $3 \%$ & $3 \%$ & Same with std. \\
\hline Cephalexin & $3 \%$ & $2 \%$ & $2 \%$ & Same with std. \\
\hline Enrofloxacin & $2 \%$ & $2 \%$ & $2 \%$ & Same with std. \\
\hline Oxytetracycline & $4 \%$ & $2 \%$ & $2 \%$ & Same with std. \\
\hline Gentamicin & $0 \%$ & $0 \%$ & $0 \%$ & Same with std. \\
\hline Neomycin & $0 \%$ & $0 \%$ & $0 \%$ & Same with std. \\
\hline
\end{tabular}

Screening of antibiotics residues were done by TLC. Samples were collected from different farms and markets in Dinajpur, Bangladesh. \% was calculated on the basis of proportion of positive and negative samples of specified antibiotics by Rf value. Std. =Standard. 


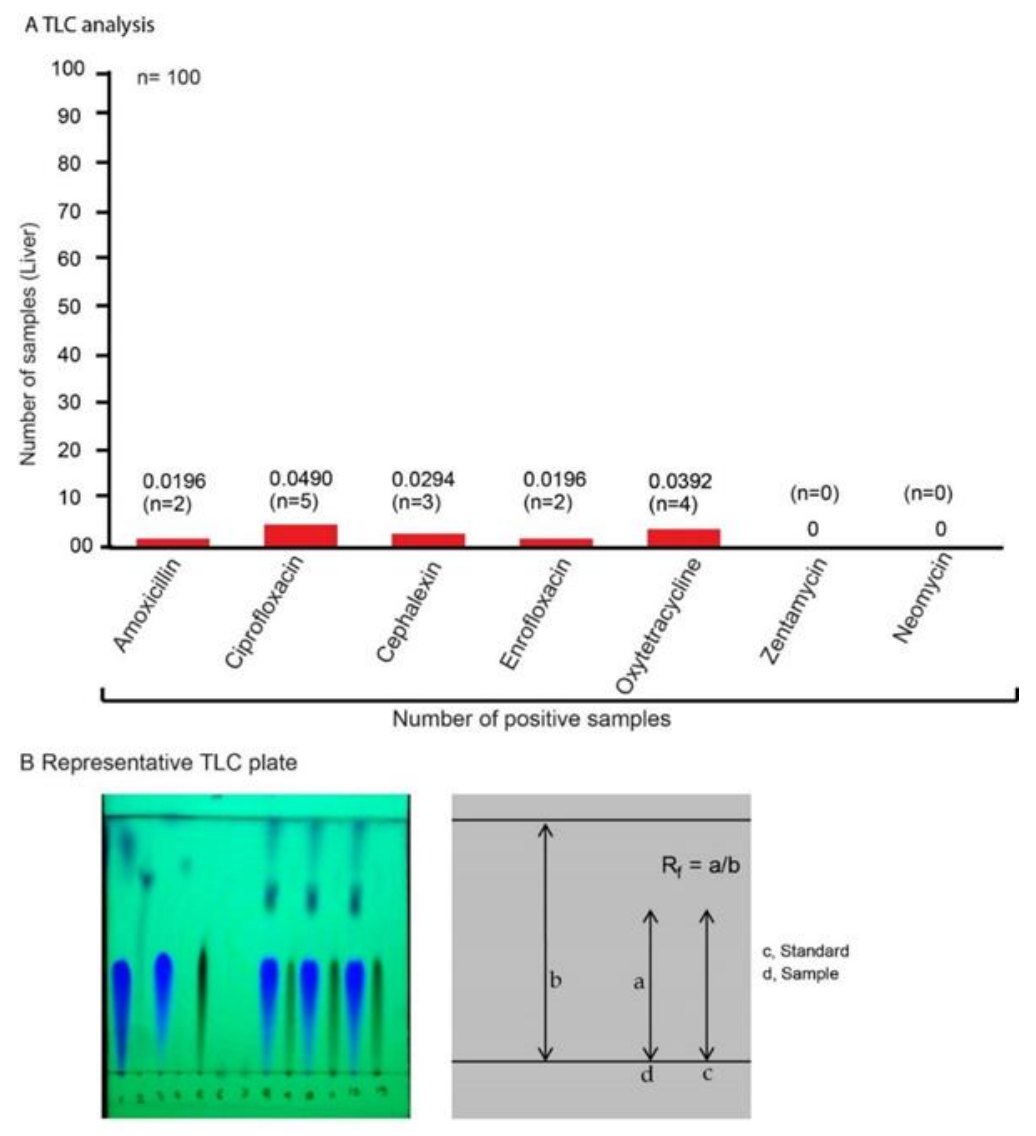

Figure 3 Antibiotics residues in liver. A, demonstrated number of positive samples for antibiotic amoxicillin, ciprofloxacin, cephalexin, enrofloxacin, oxytetracycline, gentamicin and neomycin. B, represented the TLC analysis plate and detection method of specific antibiotic residues. $n=100$.

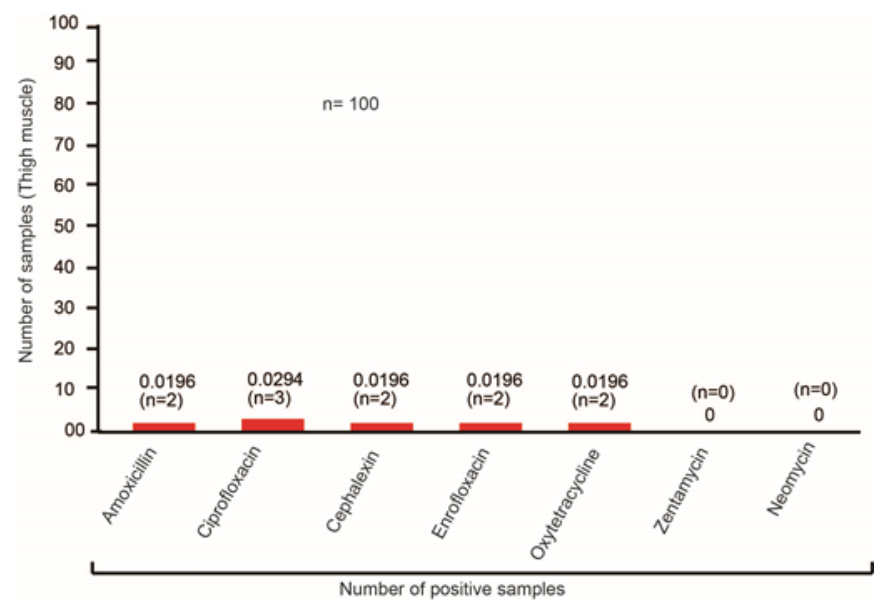

Figure 4 Antibiotics residues in thigh muscle. Number of positive samples for antibiotic amoxicillin, ciprofloxacin, cephalexin, enrofloxacin, oxytetracycline, gentamicin and neomycin are shown. $n=100$. 


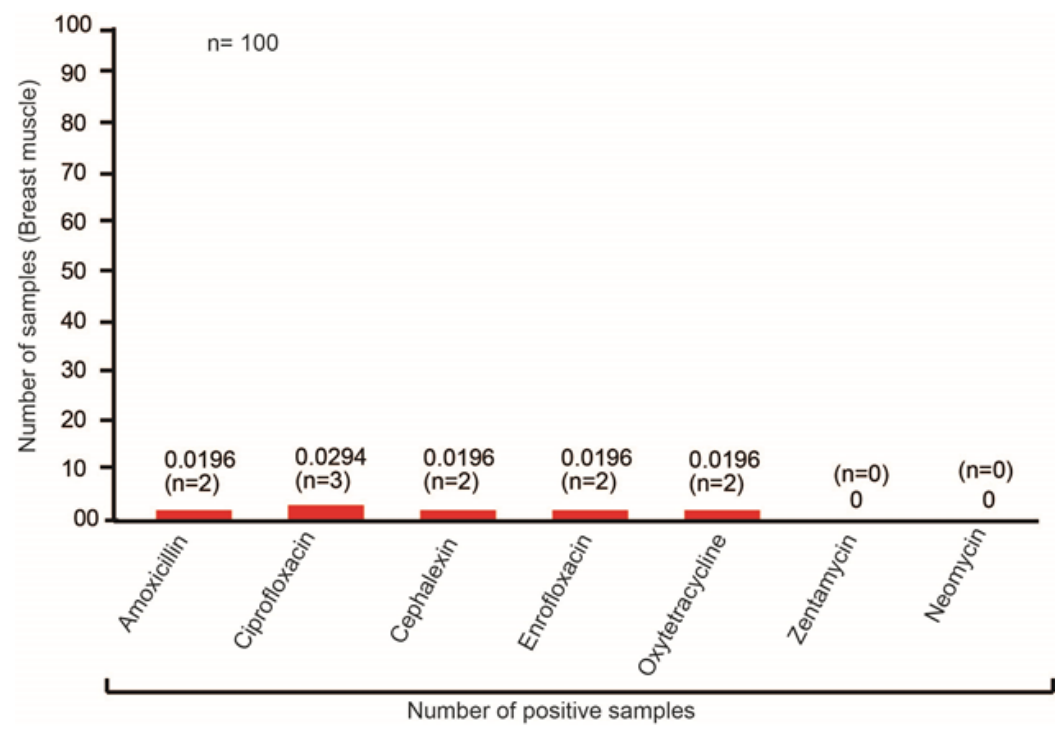

Figure 5 Antibiotics residues in breast muscle. Number of positive samples for antibiotic amoxicillin, ciprofloxacin, cephalexin, enrofloxacin, oxytetracycline, gentamicin and neomycin are shown. $\mathrm{n}=100$.

Table 2 Ciprofloxacin Antibiotic residue.

\begin{tabular}{|l|l|l|l|l|}
\hline Name of samples & $\begin{array}{l}\text { Liver, n=6 for } \\
\text { each group }\end{array}$ & $\begin{array}{l}\text { Thigh } \\
\text { muscles n=6 } \\
\text { for each } \\
\text { group }\end{array}$ & $\begin{array}{l}\text { Breast } \\
\text { muscles n=6 } \\
\text { for each } \\
\text { group }\end{array}$ & \multirow{2}{*}{} \\
\cline { 2 - 4 } & Prevalence $\%$ & Prevalence $\%$ & Prevalence $\%$ & \\
\hline Control group & $0 \%$ & $0 \%$ & $0 \%$ & No band \\
\hline Discriminate group & $0 \%$ & $0 \%$ & $0 \%$ & No band \\
\hline Indiscriminate group & $100 \%$ & $66.66 \%$ & $66.66 \%$ & Same as std. \\
\hline
\end{tabular}

Analysis of ciprofloxacin antibiotic residues was done by TLC. Samples were collected from control, discriminate and indiscriminate groups. \% was calculated on the basis of proportion of positive and negative samples of specified antibiotics by Rf value. Std. =Standard

Table 3 Oxytetracycline Antibiotic residue.

\begin{tabular}{|l|l|l|l|l|}
\hline Name of samples & $\begin{array}{l}\text { Liver, n=6 } \\
\text { for each } \\
\text { group }\end{array}$ & $\begin{array}{l}\text { Thigh } \\
\text { muscles n=6 } \\
\text { for each } \\
\text { group }\end{array}$ & $\begin{array}{l}\text { Breast } \\
\text { muscles n=6 } \\
\text { for each } \\
\text { group }\end{array}$ & \multirow{2}{\text{Rfvalue}}{} \\
\cline { 2 - 4 } & Prevalence $\%$ & Prevalence $\%$ & Prevalence $\%$ & \\
\hline Control group & $0 \%$ & $0 \%$ & $0 \%$ & No band \\
\hline Discriminate group & $0 \%$ & $0 \%$ & $0 \%$ & No band \\
\hline Indiscriminate group & $83.33 \%$ & $66.66 \%$ & $66.66 \%$ & Same as standard \\
\hline
\end{tabular}

Analysis of oxytetracycline antibiotic residues was done by TLC. Samples were collected from control, discriminate and indiscriminate groups. \% was calculated on the basis of proportion of positive and negative samples of specified antibiotics by Rf value. Std. =Standard. 

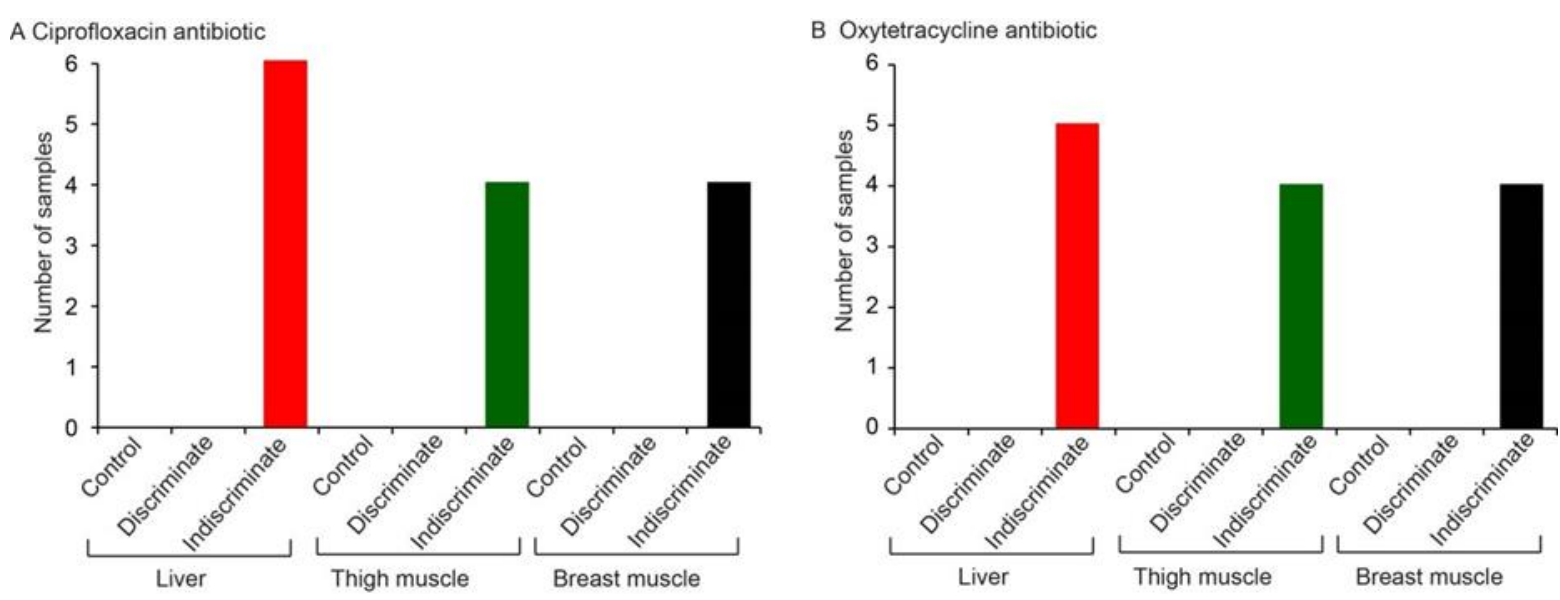

Figure 6 Antibiotics residues in liver, thigh muscle and breast muscle. Number of positive samples for antibiotic ciprofloxacin and oxytetracycline are shown. $n=108$.

\section{Discussion}

Antimicrobial resistance is becoming a burning question due to indiscriminate use of antibiotics imposing greater risk to human health. In Bangladesh, Large number of protein is supplied from poultry meat. But in poultry industry, farmers use antibiotic as therapeutic as well as growth promoter without maintaining proper withdrawal period resulting antimicrobial resistance [5].

A survey work was undertaken at the north area of Bangladesh to make aware of the people concerned with the poultry farming systems. Awareness campaigns were monitored with theme of indiscriminate use of antibiotics in poultry industries having human health hazards. All poultry farmers were found highly conscious about the danger of antibiotics residues for human consumption. Farmers in the surveyed areas confessed they don't use antibiotics after 20 days of age of broiler and if use they maintain withdrawal period before selling for human consumption. Moreover, they shared their practical knowledge on broiler rearing and mentioned that after/or at the age of 20 days of broiler birds, they don't need any antibiotics treatment. Most of the antibiotics they use at the early life of broiler as disease prevalence in broiler are more before the age of twenty days. After examining the liver, thigh and breast muscle sample using TLC, out of one hundred liver samples only two samples were positive for amoxicillin, three samples were positive for ciprofloxacin, four samples were positive for cephalexin, two samples were positive for enrofloxacin, two samples were positive for tetracycline and gentamicin \& neomycin were found negative for any samples. In case of thigh muscles, out of one hundred samples, two samples were positive for amoxicillin, three samples were positive for ciprofloxacin, four samples were positive for cephalexin, two samples were positive for enrofloxacin, two samples were positive for tetracycline and gentamycin \& neomycin were found negative for any samples. In case of breast muscles, out of one hundred samples, two samples were positive for amoxicillin, three samples were positive for ciprofloxacin, four samples were positive for cephalexin, two samples were positive for enrofloxacin, two samples were positive for tetracycline and gentamicin \& neomycin were found negative for any samples.

In our present study we also sought to find antibiotic residues in indoor control, indiscriminate and discriminate antibiotic group with proper withdrawal period. In control and discriminate group no prevalence of antibiotic found, whereas in indiscriminate group $(n=6)$ prevalence rate in liver samples were $83.33 \%$, thigh muscle samples $66.66 \%$ and breast muscle samples $66.66 \%$ found, which is similar to the research finding of ferdous et al. [5], where they found almost no prevalence of oxytetracycline residue in control and discriminate group with proper withdrawal period but prevalence rate was almost $100 \%$ in indiscriminate antibiotic group, supports our research finding. The finding is also similar to Ali et al. [1], who reported that indiscriminate antibiotic group intensity of residues for liver, kidney, spleen, thigh muscle and breast muscle samples were found $50.67 \%, 50.33 \%, 39.50 \%$ and $48.17 \%$ respectively, which is also similar to our research finding. Islam MS et al. [7] also reported that in a study of application of amoxicillin antibiotic in control, discriminate and indiscriminate group of broiler, the amoxicillin intensity in liver, kidney, thigh muscle and breast muscle were positive by TLC as $57.82 \%, 52.30 \%, 45.18 \%$ and $49.96 \%$ respectively for indiscriminate group, which is also similar to our finding. 


\section{Conclusion}

This study was conducted in the department of Pharmacology, Bangladesh Agricultural University, Mymensingh to evaluate the antibiotics residues in edible poultry tissues in broiler. A preliminary survey was conducted on poultry farms in the north area of Bangladesh with the theme of awareness against illegal or indiscriminate use of antibiotics in poultry farms. Farmers were found very aware about the indiscriminate use of antibiotics and about the human health hazards of antibiotics residues. Collected samples were investigated and only negligible number of samples was found for antibiotics residues by TLC analysis. However, discriminate and indiscriminate use of antibiotics in broilers was further examined by TLC technique. Both control and discriminate birds were found negative for any antibiotics residues by TLC analysis. On the other hand, in indiscriminate antibiotics birds, both ciprofloxacin and oxytetracycline antibiotics residues were found highly positive in liver, thigh muscle and breast muscle by TLC analysis.

The present research findings recommend that poultry farmers in north area particularly in Dinajpur district judiciously used antibiotics and poultry meat are safe for human consumption.

\section{Compliance with ethical standards}

\section{Acknowledgments}

We greatly acknowledge the support provided by the University Grants Commission, Bangladesh, Ref. No. (Project No. 2019/830/UGC).

\section{Disclosure of conflict of interest}

The authors state no conflict of interest.

\section{References}

[1] Ali MR, Sikder MH, Islam MS and Islam MS. (2020). Investigation of discriminate and indiscriminate use of doxycycline in broiler: an indoor research on antibiotic doxycycline residue study in edible poultry tissue. Asian Journal of Medical and Biological Research, 6(1), 1-7

[2] Apata DF. (2009). Antibiotic Resistance in Poultry. International Journal of Poultry Science, 8(4), 404-408.

[3] Dipeolu MA. (2004). Problems and prospects of antibiotics residues in meat products in Nigeria. Vom Journal of Veterinary Science, 1(1), 63-67.

[4] Donoghue DJ. (2003). Antibiotic residues in poultry tissues and eggs: Human health concerns? Poultry Science Journal, 82, 618-621.

[5] Ferdous MRA, Ahmed MR, Khan SH, Mukta MA, Anika TT, Hossain MT, Islam MZ and Rafiq K. (2020). Effect of discriminate and indiscriminate use of oxytetracycline on residual status in broiler soft tissues. Veterinary World, 13(1), 61-67.

[6] Geidam YA, Usman H, Musa HI, Anosike F and Adeyemi Y. (2009). Oxytetracycline and Procain Penicillin residues in tissues of slaughtered cattle in Maiduguri, Borno state, Nigeria. Terrestrial and Aquatic Environmental Toxicology, 3(2), 68-70.

[7] Islam MS, Islam Mz and Islam MS. (2019). Discriminate and indiscriminate use of amoxicillin antibiotic and detection of its residue in poultry edible tissue by thin layer chromatography (TLC) method. Asian-Australasian Journal of Food Safety and Security, 3(2), 96-102.

[8] Kukanich B, Gehring R, Webb AI, Craigmill AL and Riviere JE. (2005). Effect of Formulation and route of administration on tissue residues and withdrawal times. Journal of the American Veterinary Medical Association, $227,1574-1577$.

[9] Kumar S, Jyotirmayee K and Sarangi M. (2013). Thin Layer Chromatography: A Tool of Biotechnology for Isolation of Bioactive Compounds from Medicinal Plants. International Journal of Pharmaceutical Sciences Review and Research, 18(1), 126-132.

[10] Moats WA. (1985). Chromatographic methods for determination of macrolide antibiotic residue in tissues and milk of food producing animals. Association of Official Analytical Chemists, 68(5), 980-984. 
[11] Muhammed F, Aktar M, Rahman ZU, Javed I and Anwar MI. (2009). Role of veterinarians in providing Residuefree veterinary food. Pakistan Veterinary Journal, 29(1), 42-46.

[12] Nisha AR. (2008). Antibiotic residues: A global health hazard. Veterinary World, 1(12), 375-377

[13] Shareef AM, Jamal ZT and Yonis KM. (2009). Detection of antibiotic residues in stored poultry products. Iraqi Journal of Veterinary Sciences, 23, 45-48.

[14] Olatoye IO and Ehinwomo AA. (2011). Oxytetracycline Residues in Edible tissues of cattle slaughtered in Akure, Nigeria. Nigerian Veterinary Journal, 11, 62-66.

[15] Simon AH and Baxter GA. (2006). Biosensor screening for veterinary drug residues in food stuffs. Journal of AOAC International, 89(3), 862-867. 